\title{
Stroboscopic Images of Streamers Through Air and Over Dielectric Surfaces
}

\author{
Dirk J. M. Trienekens, Sander Nijdam, and Ute Ebert
}

\begin{abstract}
We study the propagation of streamer discharges through air and along an epoxy rod with an ICCD camera. We use stroboscopic imaging at frequencies up to $110 \mathrm{MHz}$ to visualize discharge evolution and to calculate velocities. Initial results show that surface streamers along a dielectric surface can be up to twice as fast as streamers through bulk air.
\end{abstract}

Index Terms-Imaging, surface discharges.

$\mathbf{I}$ N HIGH voltage devices, breakdown may occur because discharges seem to move more easily over insulating surfaces than through bulk gas [1]. In this paper, we use stroboscopic imaging to study these surface discharges.

To generate discharges, a positive high voltage pulse between 10 and $17 \mathrm{kV}$ [see Fig. 1(f)] is supplied by a charged capacitor that discharges after a spark gap is triggered to a needle placed $16 \mathrm{~cm}$ above a grounded cathode plane. A stainless steel sample holder containing an epoxy rod (4 $\mathrm{mm}$ in diameter) is placed on the cathode plane, in such a way that the top of this rod is $6 \mathrm{~mm}$ below and $35 \mathrm{~mm}$ to the side of the needle. Our experiments were performed in air, at pressures between 40 and 100 mbar.

To image the discharge, we use a LaVision PicoStar HR12 camera system. We send a pulse train of tunable length and frequency to the intensifier. This way, we are able to perform stroboscopic imaging of the discharge. By controlling the number of pulses and the frequency, we are able to control the number of gating cycles and the time between these gates. By measuring the monitor output of the intensifier, we are able to relate the timing of the camera to the voltage pulse [see Fig. 1(f)].

With stroboscopic imaging, we are able to study the spatial and temporal evolution of the discharge in two dimensions. Due to the stochastic behavior of the discharges it is not possible to compare different discharges, and although a streak camera would allow us to study the spatial and temporal evolution of the discharge, this would only yield accurate results for discharges that propagate in a well-defined direction. For the

Manuscript received November 3, 2013; revised January 29, 2014 and April 25, 2014; accepted June 23, 2014. Date of publication July 15, 2014 date of current version October 21, 2014. This research is supported by ABB and the Dutch Technology Foundation STW, which is part of the Netherlands Organisation for Scientific Research (NWO), and which is partly funded by the Ministry of Economic Affairs.

D. J. M. Trienekens and S. Nijdam are with the Department of Physics, Eindhoven University of Technology, Eindhoven 5612 AZ, The Netherlands (e-mail: d.j.m.trienekens@tue.nl; s.nijdam@tue.nl).

U. Ebert is with CWI Amsterdam, Amsterdam 1098 XG, The Netherlands (e-mail: ute.ebert@cwi.nl).

Color versions of one or more of the figures in this paper are available online at http://ieeexplore.iee.org.

Digital Object Identifier 10.1109/TPS.2014.2335615 highly nonreproducible discharges that propagate in multiple directions that we are studying, stroboscopic imaging yields the best results.

Pancheshnyi et al. [2] have used the same technique to study streamers, however, in their experiment the number of gating cycles was limited to 6 due to the high velocity of the discharge and the narrow gap. In our experiment, because of the lower discharge velocity and larger gap we can use up to 20 gating cycles, allowing a more detailed study of discharge phenomena like branching and the inception cloud.

Fig. 1(a)-(c) shows the images obtained at $50-\mathrm{MHz}$ intensifier gating. The needle and the contour of the dielectric rod are marked in white. The images clearly show the intermittent pattern caused by the stroboscopic gating. Three parts of the discharge can be distinguished: 1) the inception cloud [3], [4]; 2) the bulk gas streamers; and 3) the surface discharge propagating along the epoxy rod. The number of gating cycles is chosen so that the intensifier has zero gain when the discharge reaches the cathode, to prevent the imaging of secondary streamers.

Information on the inception cloud is relatively hard to derive, since the 3-D morphology of the cloud makes it harder to distinguish the maxima and minima in the intensity. To gain more information, we can reduce the gating frequency and still study the entire discharge [Fig. 1(d)], or we can zoom-in view on this part of the discharge [Fig. 1(e)].

The images show that the surface discharges are faster than the bulk gas streamers. The velocity of bulk gas streamers was determined from the longest streamers, which are assumed to have a small deviation from the vertical image plane. Under the present conditions, we find that surface discharges are typically a factor $\sim 2$ faster. Bulk gas streamers propagate at around $2 \cdot 10^{5} \mathrm{~m} / \mathrm{s}$, compared with (4-5) $10^{5} \mathrm{~m} / \mathrm{s}$ for surface streamers. Inception cloud velocities as low as $\sim 1 \cdot 10^{5} \mathrm{~m} / \mathrm{s}$ are observed just before the inception cloud breaks up into separate channels.

\section{REFERENCES}

[1] A. Sobota et al., "Speed of streamers in argon over a flat surface of a dielectric," J. Phys. D, Appl. Phys., vol. 42, no. 1, p. 015211, 2009.

[2] S. Pancheshnyi, M. Nudnova, and A. Starikovskii, "Development of a cathode-directed streamer discharge in air at different pressures: Experiment and comparison with direct numerical simulation," Phys. Rev. E, vol. 71, no. 1, pp. 16407-1-16407-12, 2005.

[3] T. M. P. Briels, E. M. van Veldhuizen, and U. Ebert, "Time resolved measurements of streamer inception in air," IEEE Trans. Plasma Sci., vol. 36, no. 4, pp. 908-909, Aug. 2008.

[4] S. Nijdam, G. Wormeester, E. M. van Veldhuizen, and U. Ebert, "Probing background ionization: Positive streamers with varying pulse repetition rate and with a radioactive admixture," J. Phys. D, Appl. Phys., vol. 44, no. 45, p. 455201, 2011. 


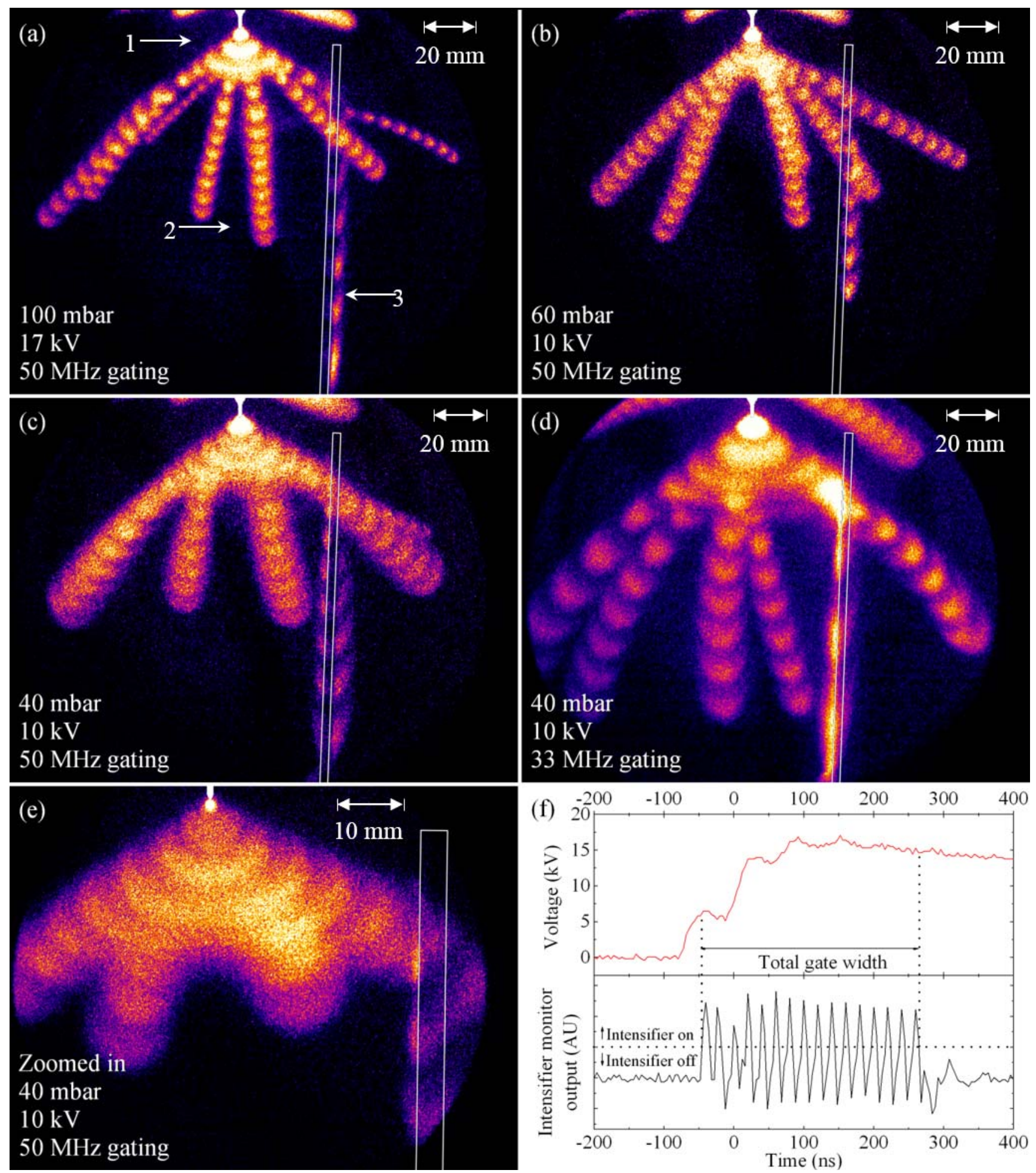

Fig. 1. Stroboscopic images of streamers through air and over dielectric surfaces in air for various pressures and voltages are presented in panels (a)-(e). The anode tip and the dielectric rod are marked in white. The repetition rate of the discharges was $3 \mathrm{~Hz}$. A typical voltage pulse and the monitor output of the intensifier are presented in panel (f). Streamers visible in the top of the image originate from the needle holder. They do not influence the streamer originating from the needle tip to the best of our knowledge. 